\title{
The solar wind plasma density control of night-time auroral particle precipitation
}

\author{
V. G. Vorobjev, B. V. Rezhenov, and O. I. Yagodkina \\ Polar Geophysical Institute, Russian Academy of Science, Apatity, Murmansk region, 184200, Russia \\ Received: 29 April 2003 - Revised: 17 October 2003 - Accepted: 4 November 2003 - Published: 19 March 2004
}

\begin{abstract}
DMSP F6 and F7 spacecraft observations of the average electron and ion energy, and energy fluxes in different night-time precipitation regions for the whole of 1986 were used to examine the precipitation features associated with solar wind density changes. It was found that during magnetic quietness $(|\mathrm{AL}|<100 \mathrm{nT})$, the enhancement of average ion fluxes was observed at least two times, along with the solar wind plasma density increase from 2 to $24 \mathrm{~cm}^{-3}$. More pronounced was the ion flux enhancement that occurred in the $b 2 i-b 4 s$ and $b 4 s-b 5$ regions, which are approximately corresponding to the statistical auroral oval and map to the magnetospheric plasma sheet tailward of the isotropy boundary. The average ion energy decrease of about $2-4 \mathrm{kev}$ was registered simultaneously with this ion flux enhancement. The results verify the occurrence of effective penetration of the solar wind plasma into the magnetospheric tail plasma sheet.
\end{abstract}

Key words. Ionosphere (auroral ionosphere, particle precipitation) - Magnetospheric physics (solar windmagnetosphere interaction)

\section{Introduction}

The problem of a solar wind particle penetration in the Earth's magnetosphere is one of the major issues in the physics of the magnetosphere. About $10^{25}-10^{26}$ particles $s^{-1}$ are required to support the observed particle concentration in the plasma sheet (Hill, 1974). However, not enough experimental data exist about particle penetration into the magnetosphere and, in particular, the physical mechanism responsible for this plasma transport and a place of particle penetration are also unknown. It is usually assumed that during the southward interplanetary magnetic field, IMF, intervals of solar wind plasma penetrate into the magnetosphere via the magnetic reconnection of the southward interplane-

Correspondence to: V. Vorobjev

(vorobjev@pgi.kolasc.net.ru) tary and geomagnetic field. During periods of northward IMF, a slow and diffusive transport of solar wind particles into the plasma sheet is assumed. A number of studies (Fairfield et al., 1981; Lennartsson and Shelley, 1986; Baumjohann et al., 1989; Lennartsson, 1992) indicated that under geomagnetic quietness the plasma sheet becomes colder and denser. Terasawa et al. (1997) carried out a statistical study of the near-Earth plasma sheet $\left(-15 R_{e}>X_{G S M}>-50 R_{e}\right)$ properties depending on solar wind parameters. It was found out that (1) during the northward IMF, the plasma sheet becomes significantly colder and denser with the solar wind plasma density increase; (2) temperatures diminish and densities increase more significantly near the dawn and dusk flanks of the plasma sheet than in the central region. They suggested that the solar wind plasma penetrates into the plasma sheet across magnetospheric flanks as a result of a slow diffusion. This paper presents the results of the statistical investigation of the relationship between the solar wind plasma density and properties (average energy and fluxes) of particle precipitating in night-time high latitudes under quiet geomagnetic conditions.

\section{Data used and results of observations}

Characteristics of precipitating ions and electrons in different auroral precipitation regions acquired by polar orbiting DMSP F6 and F7 satellites in the night sector for the whole of 1986 are examined. The notation of precipitation boundaries suggested by Newell et al. (1996) and Feldstein and Galperin (1996) is used for the investigation. In this notation the boundaries were numbered so that the number of the boundary grows with the increase in its latitudinal location. Indexes $e$ and $i$ refer to the electron and ion precipitation boundary, respectively, with $b 1$ as the "zero-energy" particle boundary. According to results by Vorobjev et al. (2000), the $b 1 e$ boundary coincides well with the equatorward boundary of diffuse auroral luminosity, and $b 2 i$ is the latitude where the energy flux of ions has the maximum. This boundary is 
(a)

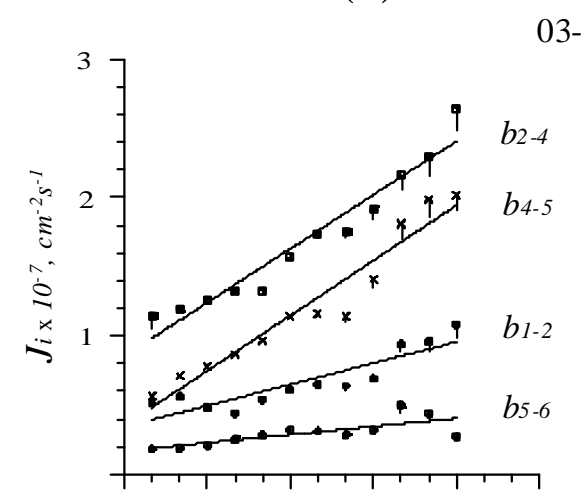

(b)

3-06 MLT

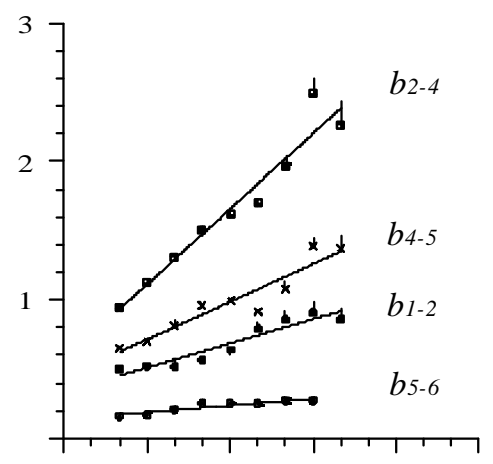

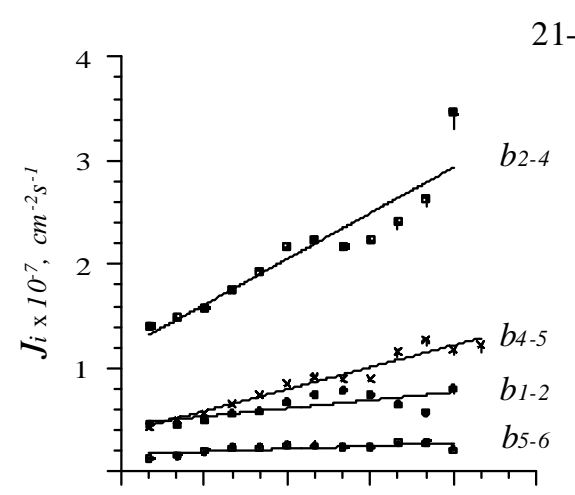

21-24 MLT
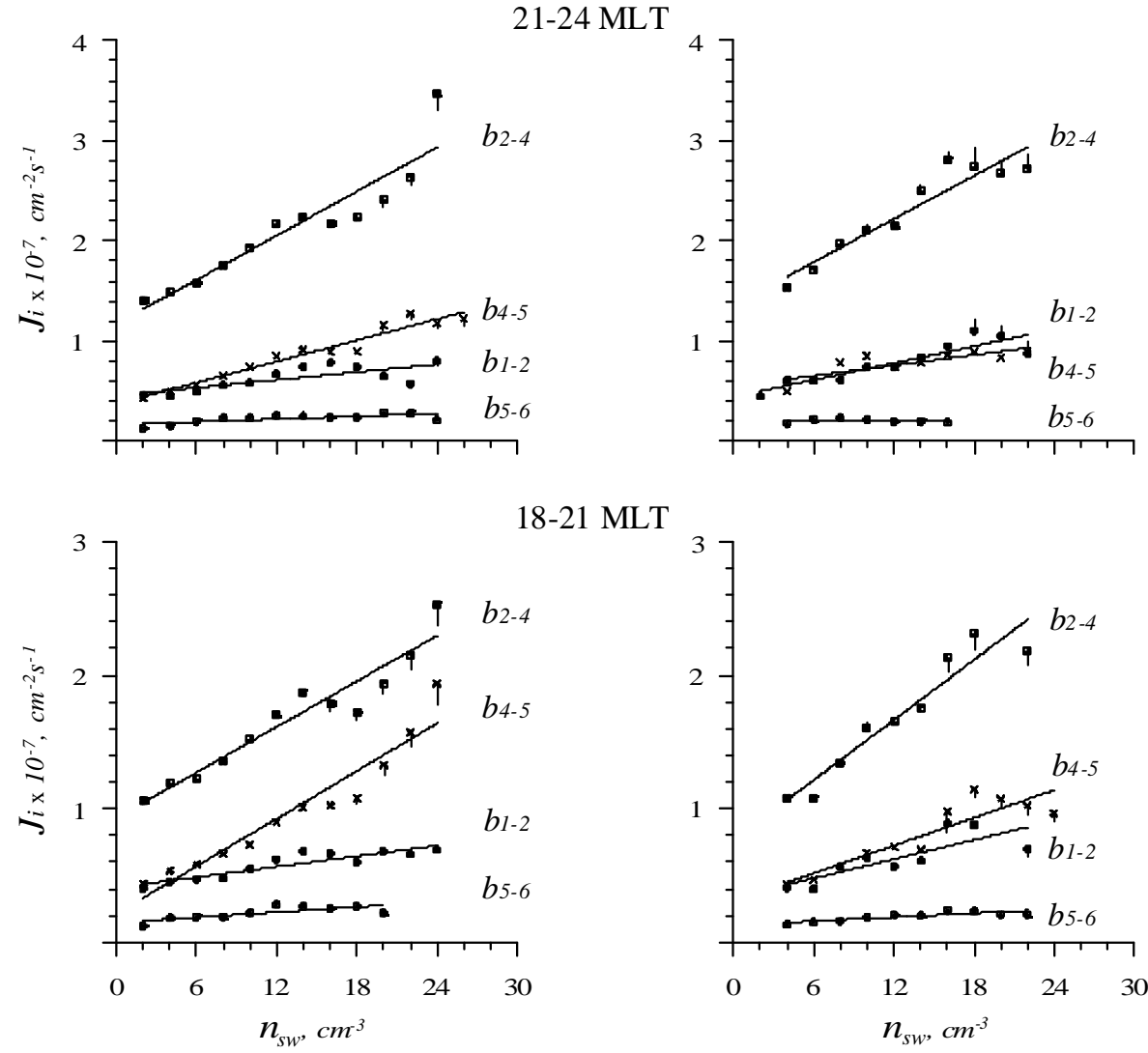

18-21 MLT

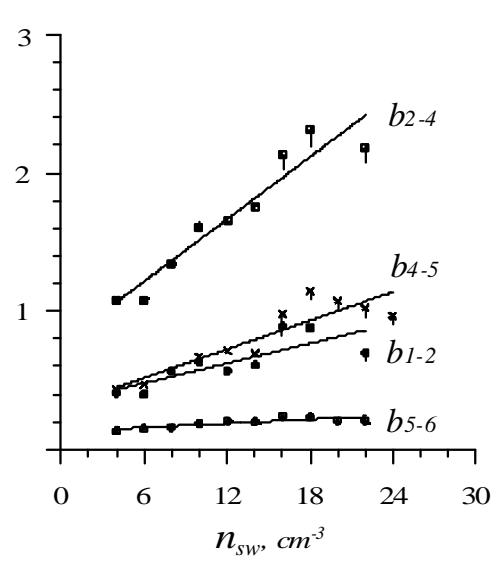

Fig. 1. Ion number flux in four precipitation regions $(b 1-b 2 i, b 2 i-b 4 s, b 4 s-b 5$ and $b 5-b 6)$ versus solar wind plasma density in the morning, pre-midnight and evening MLT sectors during periods of northward (a) and southward (b) IMF orientation.

a proxy for the ion isotropy boundary (Newell et al., 1996, 1998). Furthermore, $b 4 s$ is the equatorward boundary of spatial structured electron precipitations (low correlation coefficient between the neighboring spectra); $b 5$ is the poleward boundary where an abrupt drop by a factor of at least 4 in the precipitating energy flux is observed. According to Vorobjev et al. (2000), this boundary coincides well with the poleward boundary of the statistical auroral oval. Continuing further, $b 6$ is the poleward boundary of subvisual drizzle. It is supposed that $b 6$ often corresponds to the polar cap boundary. The average energy and the number flux of par- ticles between the adjacent precipitation boundaries in three magnetic local time sectors (03-06, 18-21 and 21-24 MLT) were determined. The 00-03 MLT sector was not investigated because of insufficient statistics owing to the orbit peculiarity of DMSP F6 and F7 satellites. The average energy and the number flux of precipitating particles were calculated within $\Delta n_{s w}=2 \mathrm{~cm}^{-3}$ solar wind density bins. Only satellite passes, where the one-hour averaged value of the AL index at the hour of auroral zone crossing and during the previous hour were $|\mathrm{AL}|<100 \mathrm{nT}$, have been selected for the analysis. The IMF and solar wind plasma data from the NSSDC 
(a)

(b)

03-06 MLT
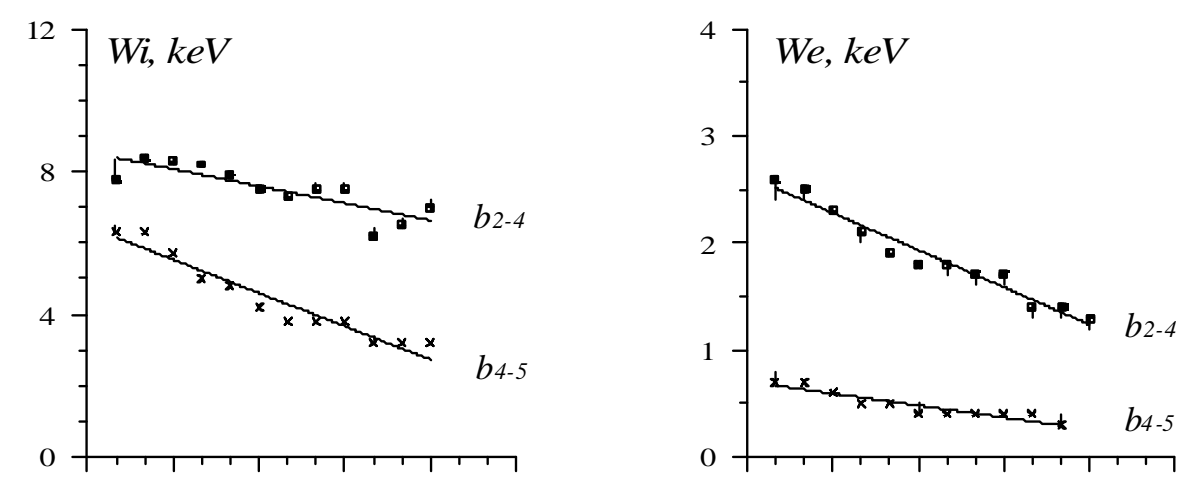

21-24 MLT
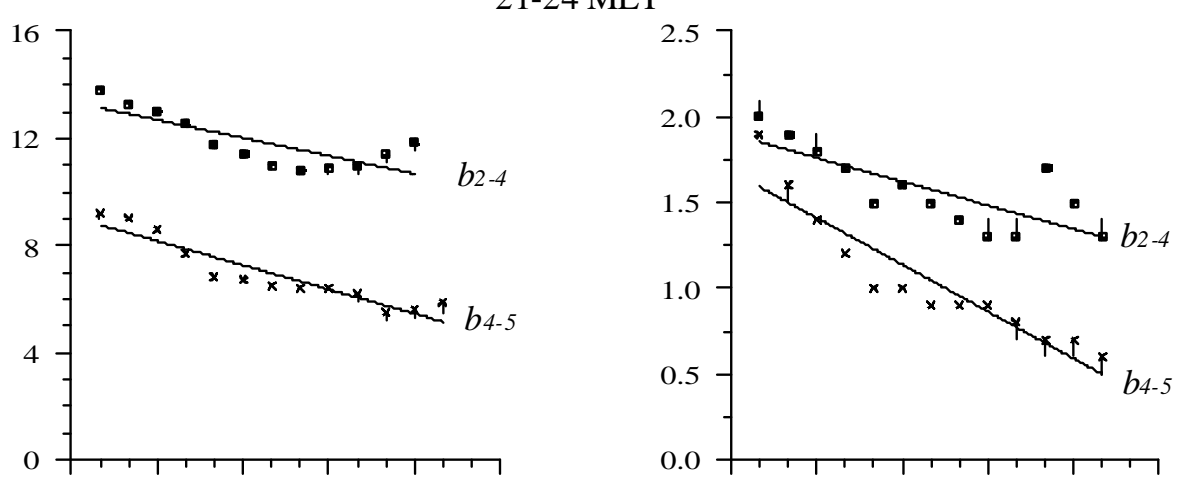

18-21 MLT
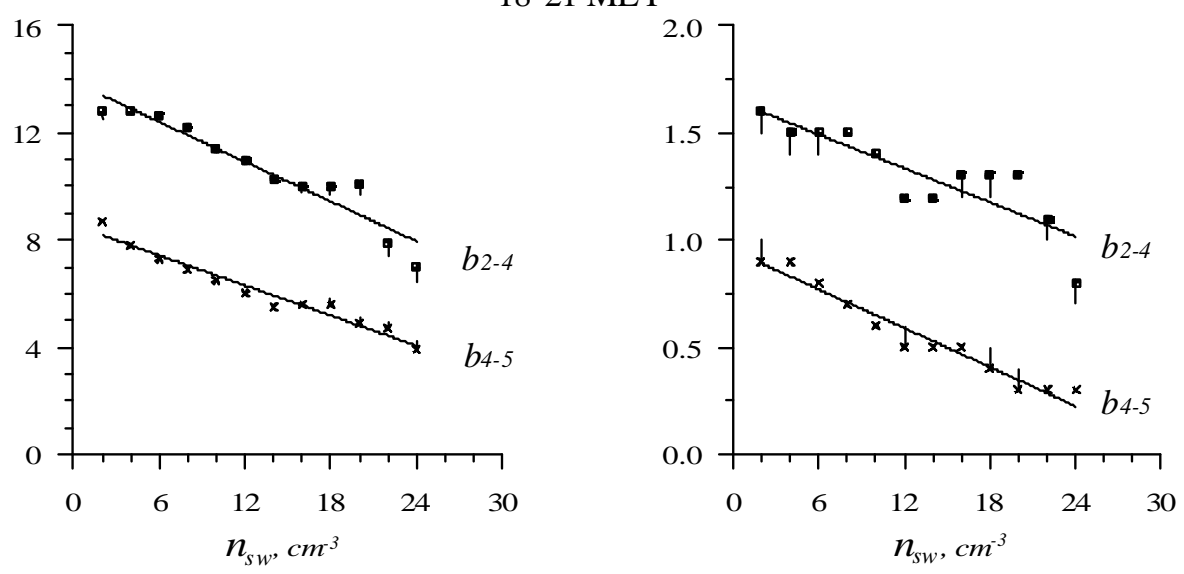

Fig. 2. Average ion (a) and electron (b) energy in the $b 2 i-b 4 s$ and $b 4 s-b 5$ regions during periods of the IMF $B_{z}>0$ versus solar wind plasma density.

OMNIWeb database in the hours of the satellite pass were used for comparison with precipitation signatures. Data sets were examined separately for the IMF $B_{z}>0$ and $B_{z}<0$ intervals. More than 3700 flights were analyzed in all. Figure 1 presents the number flux of precipitating ions in different regions versus solar wind density in the morning, premidnight and evening MLT sectors during periods of northward (a) and southward (b) IMF orientation. The vertical error bars representing a standard deviation from the mean are also provided. In order not to overload the figure, these errors are shown, where it is possible, as half of a deviation to either side. The average AL index value in all data sets was about $35 \mathrm{nT}$, and the average IMF $B_{z}$ component was equal to $2.3 \mathrm{nT}$ and $-1.2 \mathrm{nT}$ for the northward and southward IMF orientation, correspondingly. During IMF $B_{z}>0$, Fig. 1a clearly shows the enhancement of ion fluxes $\left(J_{i}, \mathrm{~cm}^{-2} s^{-1}\right)$ in all night-time MLT sectors, along with an increase in the solar wind plasma density $\left(n_{s w}, \mathrm{~cm}^{-3}\right)$. Solid lines represent 
(a)

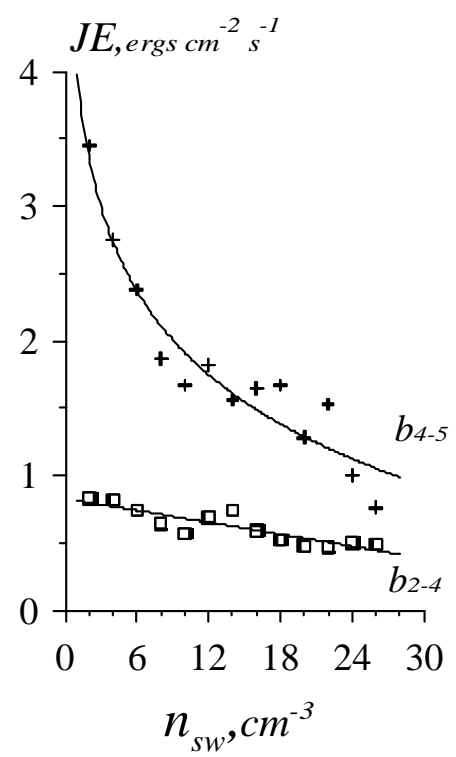

(b)

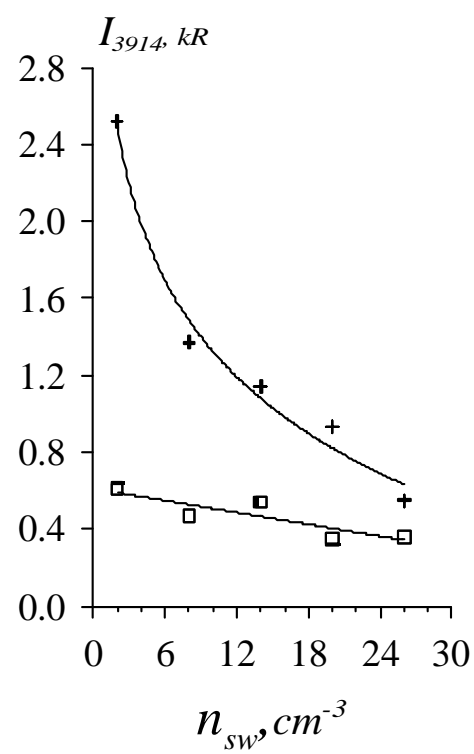

(c)

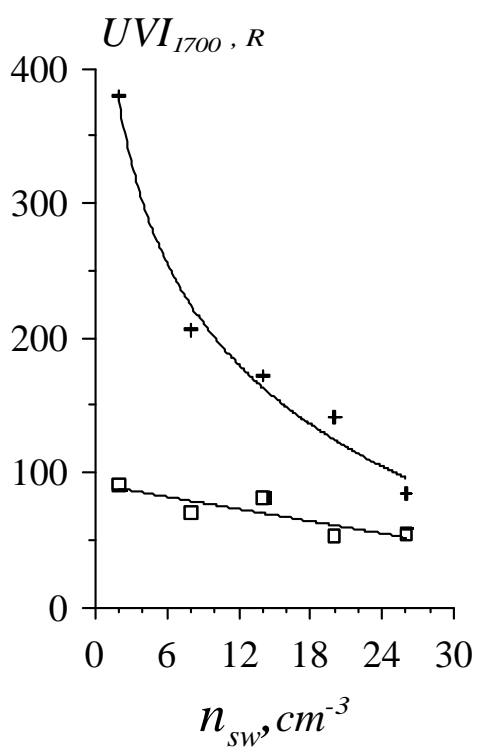

Fig. 3. Changes in the electron energy flux (a) and the calculated intensity of $391.4 \mathrm{~nm}$ (b) and LBH-long (c) bands in $b 2 i-b 4 s$ and $b 4 s-b 5$ regions in the pre-midnight sector during periods of the IMF $B_{z}>0$, depending on the solar wind plasma density.

the linear approximation of experimental points. Correlation coefficients, $r$, for all data sets are not less than 0.8. The most pronounced enhancements of the ion flux are observed in the $b 2 i-b 4 s$ and $b 4 s-b 5$ regions. In the $b 2 i-b 4 s$ region the morning sector dependence is approximated by the formula:

$$
J_{(2-4)}=\left(0.065 n_{s w}+0.85\right) 10^{7} \quad r=0.94,
$$

and in the $b 4 s-b 5$ region by the expression:

$J_{(4-5)}=\left(0.062 n_{s w}+0.43\right) 10^{7} \quad r=0.95$.

In the pre-midnight and evening sectors, respectively, the similar expressions are as follows:

$$
\begin{array}{ll}
J_{(2-4)}=\left(0.074 n_{s w}+1.17\right) 10^{7} & r=0.86 \\
J_{(4-5)}=\left(0.038 n_{s w}+0.38\right) 10^{7} & r=0.97
\end{array}
$$

and

$$
\begin{array}{ll}
J_{(2-4)}=\left(0.057 n_{s w}+0.93\right) 10^{7} & r=0.92 \\
J_{(4-5)}=\left(0.055 n_{s w}+0.25\right) 10^{7} & r=0.93 .
\end{array}
$$

The ion flux enhancement in the $b 4 s-b 5$ region looks more pronounced in the morning and in the evening, in comparison with the pre-midnight sector. In the $b 1-b 2 i$ and $b 5-b 6$ regions, the dependence of $J_{i}$ from $n_{s w}$ is expressed poorly enough in all considered MLT sectors. During the IMF $B_{z}<0$ (Fig. 1b), the most essential enhancements of the ion flux are observed in the $b 2 i-b 4 s$ region. The flux enhancement in the $b 4 s-b 5$ region during $B_{z}<0$ does not look as significant as during $B_{z}>0$. Only a weak tendency of the electron flux to be increased is observed along with the increase in the solar wind plasma density. Average ion energies $\left(W_{i}, \mathrm{keV}\right)$ in the $b 2 i-b 4 s$ and $b 4 s-b 5$ regions during northward IMF periods versus solar wind density are shown in Fig. 2a. As it is seen from Fig. 1a and 2a, simultaneously with the number flux enhancement, the average precipitating ion energy in all MLT sectors decreases approximately by $2-4 \mathrm{keV}$ when the solar wind plasma density increases from 2 to $24 \mathrm{~cm}^{-3}$. The average energy of precipitating electrons $\left(W_{e}, \mathrm{keV}\right)$ in these regions undergoes the essential decrease as well (Fig. 2b). Under the interplanetary $B_{z}<0$ conditions some tendency towards the decrease in $W_{i}$ and $W_{e}$ with the increase in solar wind plasma density, $n_{s w}$, is observed. However, the scatter in the correlations is bigger during $B_{z}<0$, than during $B_{z}>0$, generally for precipitating electron energy. Probably, it is connected with the appearance (or increase in the number) of local electron acceleration regions.

\section{Summary and discussion}

The obtained results assume that the cold solar wind plasma effectively penetrates into the plasma sheet region, which maps along geomagnetic field lines in the $b 2 i-b 4 s$ nighttime precipitation region, both during northward and southward IMF periods. In the distant plasma sheet, which maps in the $b 4 s-b 5$ region, the solar wind plasma comes across the morning and evening flanks of the magnetosphere. We shall note that during IMF $B_{z}<0$, i.e. during the magnetic reconnection in progress, the enhancement of precipitating ion 
fluxes are about the same as during the period of the IMF $B_{z}>0$. The $b 2 i-b 4 s$ region is placed between the $b 2 i$ and $b 4 s$ boundaries. The latitude of the $b 2 i$ boundary coincides well with the position of the isotropy boundary (Newell et al., 1998) and is a good proxy for the inner edge of the magnetospheric cross tail current sheet. The $b 2 i$ and $b 5$ boundaries approximately coincide with the equatorward and poleward boundaries of the average auroral oval (Vorobjev et al., 2000).

In the new notation presented by Starkov et al. (2002), the $b 2-b 5$ region was designated as the auroral oval precipitation, $A O P$. Spatially and spectrally structured electron spectra, which can be associated with discrete aurorae, are common for the $A O P$ region in all MLT sectors. Thus, the $b 2 i-b 4 s$ region approximately corresponds to the equatorial part of the auroral oval and the $b 4 s-b 5$ region to its poleward part. The $b 1-b 2$ region, called by Starkov et al. (2002) the diffuse auroral zone, $D A Z$, coincides well with the diffuse auroral luminosity equatorward of the auroral oval precipitation. To the pole of the $A O P$, between the $b 5$ and $b 6$ boundaries, there is a soft diffuse precipitation region, $S D P$, which is associated with a weak diffuse luminosity (generally in the $630.0 \mathrm{~nm}$ emission) poleward of the auroral oval. The relative position of different precipitation boundaries during the magnetic quiet periods and their dynamics during the substorm development are presented by Vorobjev et al. (2003). The latitudinal width of the $A O P$ region during the period of magnetic quietness makes $3^{\circ}-4^{\circ}$, and its equatorial part, $b 2 i-b 4 s$, is only about $1^{\circ}$ of the latitude. The pronounced decrease in the average electron energy observed with the increase in the solar wind plasma density should lead to significant changes in the auroral luminosity inside the auroral oval.

Figure 3a shows the behavior of electron energy fluxes $\left(J E\right.$, erg cm $\left.\mathrm{cm}^{-2} s^{-1}\right)$ in both the $b 2 i-b 4 s$ and $b 4 s-b 5$ premidnight regions during northward IMF periods versus solar wind plasma density. Since the electron number flux actually does not depend on the solar wind plasma density, the shape of curves in Fig. 3a are defined mainly by changes in the electron average energy. As it can be seen from the figure, the most essential changes in the energy flux are observed in the $b 4 s-b 5$ region which makes up the basic part of the average auroral oval. The intensity of a few auroral emissions is approximately proportional to precipitating electron energy flux. Figure $3 b$ illustrates the expected changes in the $1 \mathrm{NG} N_{2}^{+}$band $391.4 \mathrm{~nm}$ intensity in the $b 2 i-b 4 s$ and $b 4 s-b 5$ zones during the solar wind density increase. For the conversion of precipitating electron energy flux in intensity of the $391.4 \mathrm{~nm}$ auroral emission it was accepted, according to Dalgarno et al. (1965) that the flux of $1 \mathrm{erg} \mathrm{cm}^{-2} \mathrm{~s}^{-1}$ causes about 730 Rayleighs (R) of luminosity. In Fig. 3c the corresponding changes in the Lyman-Birge-Hopfield band intensity at about $170.0 \mathrm{~nm}$ (LBH-long) are shown, which could be registered by the UVI Imager (Torr et al., 1995) on board the Po-

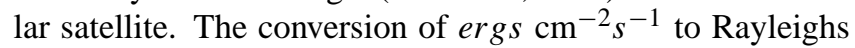
for the LBH-long band has been modeled by Germany et al. (1997) and is given as 1 erg cm$~^{-2} s-1 \approx 110 R$. Figures $3 \mathrm{~b}$ and c show that the intensity of both auroral bands in the $b 4 s-$ $b 5$ region, which makes up the basic part of the nightside auroral oval, decreased 3-4 times in the solar wind density increase from 2 to $26 \mathrm{~cm}^{-3}$. The intensity of [OI] $557.7 \mathrm{~nm}$ and $630.0 \mathrm{~nm}$ auroral emissions is substantially determined by the chemical composition of the upper atmosphere, which makes it extremely difficult to calculate their intensity. However, the average electron energy behavior allows one to estimate roughly the change in the auroral intensity $I_{6300} / I_{5577}$ ratio. According to the Judge (1972) model, simultaneously with the solar wind plasma density increases, it is possible to expect the enhancement of this ratio from approximately 0.5 up to 2 , which corresponds to the occurrence of red aurorae of rather significant intensity. These conclusions allow one to explain naturally the results received earlier by Liou et al. (1998) and Khviusova and Leontyev (2002). Liou et al. (1998) used the Polar UVI images at the LBH-long band for a correlative study of the energy deposition rate with the concurrent solar wind plasma and IMF observations. They showed (their Fig. 5e) that the midnight aurora had a trend of decreasing in power with the solar wind density increase. Khviusova and Leontyev (2002) have found out that during the magnetic quietness, the large $(\geq 5 k R)$ intensities of the red oxygen emission $(630.0 \mathrm{~nm})$ were observed in the night-time aurorae generally during periods with an increased value $\left(n_{s w}>10 \mathrm{~cm}^{-3}\right)$ of solar wind plasma density.

According to the results of our investigation, the average electron energy in the auroral oval decreases essentially with the solar wind plasma density enhancement. It should lead to the decrease in the auroral luminosity in the UVI region, as well as in the main $391.4 \mathrm{~nm}, 427.8 \mathrm{~nm}$ and $557.7 \mathrm{~nm}$ auroral emissions, simultaneously with the enhancement of the $630.0 \mathrm{~nm}$ luminosity, which is caused by lower energy electron precipitation, i.e. the green aurorae are replaced with the red ones. What is the reason for the sharp drop in the $b 4 s-$ $b 5$ region electron energy flux, while the solar wind density increases? It is possible to assume, that even under quiet geomagnetic conditions and the IMF $B_{z}>0$, there are local electron acceleration regions in the auroral oval near midnight (weak auroral arcs and diffuse band).

Figure 2 shows that during the low solar wind plasma density, $n_{s w}$, the average electron energy is higher in 21-24 MLT than in 18-21 MLT and 03-06 MLT. The decrease in the average electron energy, along with the increase in the $n_{s w}$ results in the reduction of E-region ionospheric conductivity and suppression of electron field-aligned acceleration mechanisms (disappearance of discrete aurorae). One can see in Fig. 2, the average electron energy in the $b 4 s-b 5$ region is very low and approximately identical in all night-time sectors under the large solar wind plasma density conditions (no accelerations around midnight). So, two factors can be important: (1) the cooling of the magnetospheric plasma (a decreasing in the precipitation particle average energy) due to solar wind plasma penetration into the magnetosphere and (2) simultaneous suppression of electron acceleration mechanisms. Figure 1 shows for the most part a factor of 2 to 2.5 increase in the ion precipitation flux, while the solar wind 
density increases 12 -fold. We guess that this relation determines the efficiency factor of all mechanisms from solar wind plasma penetration into the magnetosphere to the particle precipitation.

\section{Conclusion}

Observations from DMSP F6 and F7 spacecrafts of the average electron and ion energy, and energy flux in different night-time precipitation regions were used to examine the precipitation features associated with solar wind density changes. It was found that during magnetic quiet periods $(|\mathrm{AL}|<100 \mathrm{nT})$, the enhancement of the average ion flux was observed, along with the solar wind plasma density increase. This ion flux enhancement was more pronounced in the $b 2 i-b 4 s$ and $b 4 s-b 5$ regions, which are approximately corresponding to the statistical auroral oval and map to the magnetospheric plasma sheet tailward of the isotropy boundary. The average ion energy decrease of about $2-4 \mathrm{keV}$ was registered simultaneously with this ion flux enhancement. The results verify the occurrence of effective penetration of the solar wind plasma into the magnetospheric tail plasma sheet. Since the intensity of auroral $1 \mathrm{NG} \mathrm{N}_{2}^{+}$and LymanBirge-Hopfield bands is approximately proportional to the precipitating electron energy flux, there should be expected a decrease in the auroral luminosity in the UVI spectral region and in the main auroral emissions 391.4, 427.8 and $557.7 \mathrm{~nm}$. Simultaneously, an increase in the $630.0 \mathrm{~nm}$ luminosity excited by the lower energy electron precipitation should be observed.

Acknowledgements. The DMSP particle detectors were designed by Dave Hardy of AFRL, and data obtained from JHU/APL. We thank D. Hardy, F. Rich, and P. Newell for its use. We thank Drs J.H. King and N. Papitashvili for providing IMF and solar wind plasma observations in the NSSDC OMNIWeb database. The WDC for geomagnetism in Kyoto provided magnetic activity indexes. The paper was supported by the RFBR grants 02-05-64807 and 99-0565611, INTAS-2000-752. Authors acknowledge the support from the Division of Physical Sciences of the Russian Academy of Sciences (program DPS-16).

Topical editor T. Pulkkinen thanks W. Lennartsson and another referee for their help in evaluating this paper.

\section{References}

Baumjohann, W., Paschmann, G., and Cattell, C. A.: Average plasma properties in the central plasma sheet, J. Geophys. Res., 94, 6597, 1989.

Dalgarno, A., Latimer, I. D., McConkey J. W.: Corpuscular bombardment and radiation, Planet. Space Sci., 13, 1008, 1965.

Fairfield, D. H., Lepping, R. P., Hones Jr., E. W., et al.: Simultaneous measurements of magnetotail dynamics by IMP spacecraft, J. Geophys. Res., 86, 1396, 1981.

Feldstein, Ya. I. and Galperin, Y. I.: Structure of the auroral precipitations in the nightside sector of the magnetosphere, Cosmic Research (in Russian), 34, 227, 1996.

Germany, G. F., Parks, G. K., Brittnacher, M., Cumnock, J., Lumnerzheim, D., Spann, J. F., Chen, L., Richards, P. G., Rich, F J.: Remote determination of auroral energy characteristics during substorm activity, Geophys. Res. Lett., 24, 995, 1997.

Hill, T. W.: Origin of the plasma sheet, Rev. Geophys. Space Sci., 12, 379, 1974.

Judge, R. J. R.: Electron excitation and auroral emission parameters, Planet. Space Sci., 20, 2081, 1972.

Khviusova, T. A. and Leontyev, S. V.: Solar wind density influence on the auroral red line $(630.0 \mathrm{~nm})$ intensity, Geomagnetism and Aeronomy (in Russian), 42, 4, 519, 2002.

Lennartsson, W. and Shelley, E. G.: Survey of 0.1 -to $16 \mathrm{keV} / \mathrm{e}$ plasma sheet ion composition, J. Geophys. Res., 91, 3061, 1986.

Lennartsson, W. A.: Scenario for solar wind penetration of Earth's magnetotail based on ion composition data from the ISEE1 spacecraft, J. Geophys. Res., 97, 19921, 1992.

Liou, K., Newell, P. T., Meng, C.-I., Brittnacher, M., Parks, G.: Characteristics of the solar wind controlled auroral emissions, J. Geophys. Res. 103, 17 543, 1998.

Newell, P. T., Feldstein, Y. I., Galperin, Yu. I., Meng, C.-I.: Morphology of nightside precipitation, J. Geophys. Res. 101, 10737 , 1996.

Newell, P. T., Sergeev, V. A., Bikkuzina, G. R., Wing, S.: Characterizing the state of magnetosphere: testing the ion precipitation maximal latitude (b2i) and the ion isotropy boundary, J. Geophys. Res. 103, 4739, 1998.

Starkov, G. V., Rezhenov, B. V., Vorobjev, V. G., Feldstein, Y. I., Gromova, L. I.: Structure of dayside auroral precipitation, Geomagnetism and Aeronony (in Russian), 42, 2, 186, 2002.

Terasawa, T., Fujimoto, M., Mukai, T. et al.: Solar wind control of density and temperature in the near - Earth plasma sheet WIND/GEOTAIL collaboration, Geophys. Res. Let., 24, 935, 1997.

Torr, M. R., Torr, D. G., Zukic, M., et al.: A far ultraviolet imager for the international solar-terrestrial physics mission, Space Sci. Rev., 71, 329, 1995

Vorobjev, V. G., Gromova, L. I., Rezhenov, B. V., Starkov, G. V., Feldstein, Y. I.: Variations of the boundaries of plasma precipitation and auroral luminosity in the night-time sector, Geomagnetism and Aeronony (in Russian), 40, 3, 79, 2000.

Vorobjev, V. G., Yagodkina, O. I., Starkov, G. V., Feldstein, Y. I.: Midnight electron precipitation pattern for substorm development, Ann. Geophys, 21, 2003. 